In der Rubrik "Literatur kompakt" referieren und kommentieren diese Experten für

Sie die wichtigsten Originalarbeiten aus der internationalen Fachliteratur.

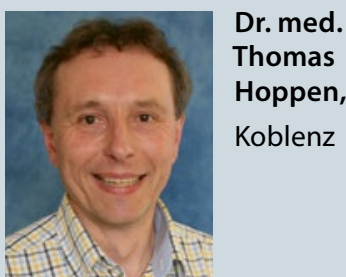

\title{
Kommt bald ein Neugeborenenscreening auf Gallengangsatresie?
}

Der durchaus längerfristige Erfolg einer Kasai-Operation ist bei frühzeitiger Diagnose einer Gallengangsatresie bekanntlich hoch. Ein interessanter Beitrag in einer aktuellen Ausgabe des New England Journal of Medicine geht der Frage nach, wie ein mögliches Screening aussehen könnte.

Cymptome einer neonatalen Cholestase sind Ikterus prolongatus, acholische Stühle und dunkel gefärbter Urin. Die gefürchtete Gallengangsatresie tritt mit einer Inzidenz von 1:10.000-19.000 auf und ist für rund $60 \%$ aller Lebertransplantationen im 1 . Lebensjahr verantwortlich. Nur die Hepatoportoenterostomie nach Kasai kann eine frühe Transplantation für einen gewissen Zeitraum verhindern. Das Outcome dieses Eingriffs ist besser, wenn er vor dem 30.-45.
Lebenstag durchgeführt wird. In den USA liegt das durchschnittliche Operationsalter allerdings bei 60-70 Tagen. Deswegen forderte die American Academy of Pediatrics zur Durchführung von Studien zur Evaluation eines Screenings auf.

Vorgestellt wird von Harpavat et al. ein zweistufiges Konzept. Aufgenommen wurden 11.636 Neugeborene (99\% aller Geburten) von vier Entbindungseinrichtungen in Housten/Texas, über einen Zeitraum von 15 Monaten. Routi-

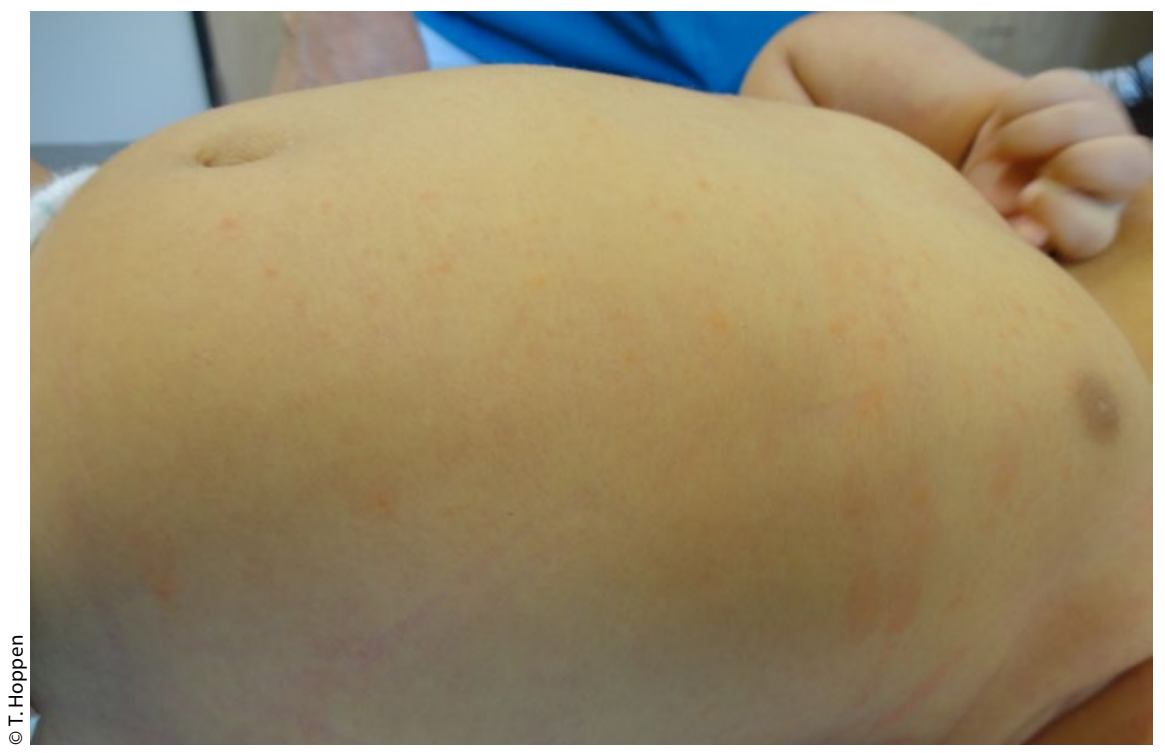

Neugeborenes mit prolongierter Hyperbilirubinämie und Hepatomegalie

nemäßig wurden bei diesen Kindern das gesamte und das konjugierte Bilirubin bestimmt. Positiv war in Stufe 1 jedes Kind mit einem direkten oder konjugierten Bilirubin über der 95. Perzentile (Abnahme $\leq 60$. Lebensstunde). In Stufe 2 wurden nur bereits in Stufe 1 positiv getestete Kinder untersucht. Positiv waren hier wiederum Kinder, wenn ihre Werte ansteigende Konzentrationen bei diesem Wiederholungstest zeigten (Zeitraum: $\leq 2$.-3. Lebenswoche). Elf Kinder wurden detektiert, davon erfuhren drei eine invasive Abklärung. Eines hatte ein Alpha-1-Antitrypsin-Problem, das zweite eine Kombination aus Gallengangsatresie mit schwerem kongenitalen Herzfehler und das dritte eine isolierte Gallengangsatresie. Interessanterweise hatte letzteres Kind keine Gelbsucht und noch normal gefärbte Stühle. Bei diesem erfolgte die Kasai-Operation bereits am 26. Lebenstag, die Konzentration des konjugierten Bilirubins normalisierte sich daraufhin innerhalb von 3 Monaten und der Patient überlebt bereits 2 Jahre transplantationsfrei.

Harpavat S et al. Newborn bilirubin screening for biliary atresia. N Engl J Med 2016; 375;605-6

\section{Kommentar}

Eine Sensitivität von $100 \%$ und eine Spezifität von $99,9 \%$ bei einem positiven Vorhersagewert von immerhin $18,2 \%$ sprechen für sich. Diese vorläufigen Ergebnisse - an einer zunächst noch sehr kleinen Patientenzahl unterstützen die Hypothese, dass ein Screening von Neugeborenen auf direktes oder konjugiertes Bilirubin eine Gallengangsatresie zu identifizieren hilft, bevor klinische Symptome auftreten.

Dr. Thomas Hoppen 